\title{
CT Angiography-Based Radiomics as a Tool for Carotid Plaque Characterization and Surgery Patient Selection: A Pilot Study
}

Savino Cilla ( $\square$ savinocilla@gmail.com )

Gemelli Molise Hospital, Università Cattolica del Sacro Cuore

Gabriella Macchia

Gemelli Molise Hospital, Università Cattolica del Sacro Cuore Jacopo Lenkowicz

Fondazione Policlinico Universitario "Agostino Gemelli" IRCCS

Elena H. Tran

Fondazione Policlinico Universitario "Agostino Gemelli" IRCCS

\section{Antonio Pierro}

"A. Cardarelli" Regional Hospital ASReM

\section{Lella Petrella}

Gemelli Molise Hospital

\section{Mara Fanelli}

Gemelli Molise Hospital

\section{Celestino Sardu}

University of Campania "Luigi Vanvitelli

\section{Alessia Re}

Gemelli Molise Hospital, Università Cattolica del Sacro Cuore

\section{Luca Boldrini}

Fondazione Policlinico Universitario "Agostino Gemelli" IRCCS

\section{Luca Indovina}

Fondazione Policlinico Universitario A. Gemelli, Università Cattolica del Sacro Cuore

\section{Carlo Maria Filippo}

Gemelli Molise Hospital, Università Cattolica del Sacro Cuore

\section{Eugenio Caradonna}

Gemelli Molise Hospital, Università Cattolica del Sacro Cuore

Francesco Deodato

Gemelli Molise Hospital, Università Cattolica del Sacro Cuore

\section{Massimo Massetti}

Fondazione Policlinico Universitario A. Gemelli, Università Cattolica del Sacro Cuore 
Fondazione Policlinico Universitario "Agostino Gemelli" IRCCS

\section{Pietro Modugno}

Gemelli Molise Hospital, Università Cattolica del Sacro Cuore

\section{Research Article}

Keywords: Radiomics, Carotid, Angiography, Plaques

Posted Date: December 9th, 2021

DOI: https://doi.org/10.21203/rs.3.rs-1123643/v1

License: (c) (1) This work is licensed under a Creative Commons Attribution 4.0 International License. Read Full License 


\section{Abstract}

Objectives: Radiomics is a quantitative method able to analyze a high-throughput extraction of minable imaging features. Herein, we aim to develop a CT angiography-based radiomics analysis and machine learning model for carotid plaques to discriminate vulnerable from no vulnerable plaques.

Methods: Thirty consecutive patients with carotid atherosclerosis were enrolled in this pilot study. At surgery, a binary classification of plaques was adopted ("hard" vs "soft"). Feature extraction was performed using the R software package Moddicom. Pairwise feature interdependencies were evaluated using the Spearman rank correlation coefficient. A univariate analysis was performed to assess the association between each feature and the plaque classification and chose top-ranked features. The feature predictive value was investigated using binary logistic regression. A stepwise backward elimination procedure was performed to minimize the Akaike information criterion (AIC). The final significant features were used to build the models for binary classification of carotid plaques, including logistic regression (LR), support vector machine (SVM), and classification and regression tree analysis (CART). All models were cross-validated using 5-fold cross validation. Class-specific accuracy, precision, recall and F-measure evaluation metrics were used to quantify classifier output quality.

Results: A total of 230 radiomics features were extracted from each plaque. Pairwise Spearman correlation between features reported a high level of correlations, with more than $80 \%$ correlating with at least one other feature at $|\rho|>0.8$. After a stepwise backward elimination procedure, the entropy and volume features were found to be the most significantly associated with the two plaque groups $(\mathrm{p}<$ 0.001 ), with AUCs of 0.92 and 0.96 , respectively. The best performance was registered by the SVM classifier with the RBF kernel, with accuracy, precision, recall and F-score equal to 86.7, 92.9, 81.3 and $86.7 \%$, respectively. The CART classification tree model for the entropy and volume features model achieved $86.7 \%$ well-classified plaques and an AUC of 0.987.

Conclusion: This pilot study highlighted the potential of CTA-based radiomics and machine learning to discriminate plaque composition. This new approach has the potential to provide a reliable method to improve risk stratification in patients with carotid atherosclerosis.

\section{Introduction}

Carotid atherosclerotic plaque ulceration and rupture are one of the main causes of major adverse cardiac events such as ischemic stroke, which account for approximately one-fourth of all strokes (1). Luminal stenosis degree has been historically considered the most important parameter to grade the severity of atherosclerotic disease and risk-stratify patients and treatment decision-making (2). However, new pathological studies have demonstrated major differences in the composition and risk of rupture for plaques with identical degrees of stenosis (3). The technological advancement of vascular imaging techniques such as CT and MR angiography allowed a routine high-resolution characterization of carotid plaque features. Recent literature has highlighted the critical role of plaque composition as a predictive 
factor of future cerebrovascular events (4-8). Plaque ulceration, intraplaque hemorrhage, thin or ruptured fibrous cap, lipid-rich necrotic core and the presence of calcification have been proposed as structural plaque characteristics to distinguish vulnerable from nonvulnerable plaques (9-12). In particular, vulnerable plaques reported a high risk of rupture, resulting in thrombus formation and embolization of plaque material and/or thrombus migrating into the intracranial circulation. Among advanced imaging modalities, CT angiography (CTA) has been established as an effective robust noninvasive imaging technique to evaluate carotid atherosclerotic plaque-related stenosis and to provide information on the composition of the plaque (13). Indeed, in addition to the stenosis degree assessment, other major relevant applications included the ability of CTA to identify specific imaging markers associated with cerebrovascular ischemia, including plaque thickness (14), ulceration (15), and calcification (16). However, despite its reliability, CTA imaging is a complex procedure requiring high expertise in assessing plaque structure components, translating into a qualitative and subjective task (17).

Radiological images, including those obtained by CTA imaging, are multidimensional data sets in which each voxel value represents a specific metric based on some physical characteristics (18). This is the specific field of interest of radiomics, a complex computing process of obtaining a large number of quantitative parameters, called "radiomic features", from these multidimensional spatial datasets. These features aim to mathematically quantify the morphological characteristics of a specific tissue, often difficult to interpret by visual assessment. In recent years, this approach has proven to be an effective tool in oncology, reporting a major ability in tumor staging and grading, response assessment to treatments and prediction of clinical outcomes (19).

Recently, a radiomics approach has emerged as a reliable tool for the assessment of cardiovascular diseases, particularly for the quantitative study of coronary and carotid atherosclerotic plaques $(20,21)$. The first attempts employed CT-based (22) and ultrasound-based (23) texture analysis to discriminate symptomatic carotid plaques reporting promising results. CT- and MRI-based machine learning algorithms were successfully implementedto accurately distinguish symptomatic from asymptomatic carotid plaques and proved to be superior to the traditional model in the identification of high-risk plaques $(22,24)$. Le et al. $(25)$ evaluated the ability of several robust features to identify culprit and nonculprit arteries using different machine learning algorithms and reported an average area under the curve of 0.73 and accuracy of $69 \%$. Concerning risk assessment, Van Assen et al. (26) aimed to investigate an Al algorithm to predict major cardiac adverse events based on plaque characteristics. The authors reported that the model obtained combining both clinical risk factors and morphological features in a logistic regression analysis provided an accuracy of $87 \%$ with an AUC of 0.924 . A recent study (27) investigated the potential of CTA texture analysis in identifying vulnerable patients with carotid artery atherosclerosis. The authors reported that texture analysis revealed a statistically significant difference in the skewness feature to distinguish symptomatic and asymptomatic patients, with an AUC of 0.68 , sensitivity of $64 \%$ and specificity of $71 \%$.

The aim of the study was to develop a CTA-based radiomics analysis model on carotid plaquesto discriminate vulnerable from nonvulnerable plaques and to identify surgical candidates in a timely 
manner. Different binary classification models were built using a machine-learning approach to improve the identification.

\section{Material And Methods Study population}

This study was conceived within the Gemelli Generator Molise (G2 M) group study and was approved by the Institutional Review Board of the Gemelli Molise Hospital (Campobasso, Italy). Thirty patients were retrospectively enrolled from October 2015 to October 2019. All patients aged $>18$ and $<75$ years, diabetic and nondiabetic, with indications to receive carotid endarterectomy (TEA) for extracranial high-grade $(>70 \%)$ internal carotid artery stenosis were included. High-risk cardiological patient candidates for carotid revascularization by stent angioplasty or myocardial and carotid revascularization by combined aorto-coronary bypass surgery and carotid TEA were excluded.

Patients entering the study signed informed consent for treatment and use of their clinical data for research or educational purposes. Patient data were anonymized, managed according to the existing legislation for the protection of privacy and uploaded to the segmentation and analysis platforms.

\section{Plaques classification at surgery}

At surgery, carotid artery plaques were considered "hard" when their composition was predominantly calcified or with collagen-rich sclerotic tissue and "soft" when they contained atheromatous debris or in case of intraplaque hemorrhage $(28,29)$. This schematic classification was based on the common assumptions that the sclerotic component, which is fibrous tissue, in "hard" plaques should stabilize these plaques and protect them against disruption, while the "soft" plaques should be considered "at-risk" because the atheromatous gruel destabilizes the plaque, thus leaving it vulnerable to rupture (30). Moreover, this binary classification choice was usefulto counteract the small sample size.

\section{Imaging data and segmentation}

CT angiography was performed with a 128-slice scanner (Brilliance 128, Philips Healthcare, Best, the Netherlands). An 18-gauge intravenous catheter was placed in the antecubital vein; $55 \mathrm{~mL}$ of contrast, iomeprol $400 \mathrm{mg} / \mathrm{ml}$ (Iomeron ${ }^{\circledR}$, Bracco, Milan, Italy) was infused at $4 \mathrm{~mL} / \mathrm{s}$ after an initial injection delay depending on an attenuation of $140 \mathrm{HU}$ in the ascending aorta with a slice thickness of $0.9 \mathrm{~mm}$. Curved multiplanar and volume rendering reconstructions were obtained by means of dedicated computer software.

CTA images were transferred by the Digital Imaging and Communications in Medicine (DICOM) protocol to a treatment planning system (Philips Pinnacle, Fichburg, USA) in our radiation oncology department, containing a dedicated module for image segmentation and analysis. The plaques were manually segmented on all CT slices by a senior radiologist (A.P.) and by a vascular surgeon (P.M.).

\section{Radiomics analysis}


Feature extraction was performed using Moddicom (31), an R software package composed of classes written using functions and the $\mathrm{S} 3$ objects paradigm, which was standardized through the Image Biomarker Standardization Initiative (IBSI) (32). Two hundred thirty features, including first-order statistical features, shape-based features, gray-level cooccurrence matrix features (GLCM), gray-level run length matrix (GLRLM), gray-level size zone matrix (GLSZM), neighboring gray tone difference matrix (NGTDM), and gray level dependence matrix (GLDM) features, were extracted (32).

\section{Features selection and modeling}

Radiomic feature extraction usually leads to a highly redundant feature space. Since many of these features could be highly correlated with one another, a feature selection method is necessary to avoid collinearity and reduce dimensionality. Following the common practice known as "one in ten rule", i.e., for each feature, at least ten subjects should be available for each class, our aim was to select no more than two features to build reliable models. To address this problem, we used a multistage feature selection method. First, the pairwise feature interdependencies were evaluated using the Spearman rank correlation coefficient $(\rho)$, aiming to identify complex functional dependencies between features. The redundant features, defined as those having $|\rho| \geq 0.8$, were eliminated. In the second stage, a univariate analysis was performed to assess the association between each feature and the plaque classification and chose the top-ranked features. Subsequently, we investigated the feature predictive value in a binary logistic regression. To determine the relative importance of features, the remaining features were included in a stepwise backward elimination approach. In each step of this method, a feature is considered for subtraction from the set of explanatory variables based on specified criteria, including the Akaike information criterion (AIC). In particular, we tested the impact of the deletion of each feature whose loss gives the most statistically insignificant deterioration on the model. AIC is derived from information theory and is a model selection criterion that penalizes models for which adding new explanatory variables does not supply sufficient information to the model. The aim is to minimize the AIC. It is defined as:

$$
A I C=2 \log L(M)+2 K
$$

where $\log L(M)$ is the maximized log likelihood for the fitted model, $N$ is the sample size and $K$ is the number of covariates including an intercept.

The final significant features were finally used to build the models for binary classification of carotid plaques, including logistic regression (LR), support vector machine (SVM), and classification and regression tree analysis (CART).

LR is a classical machine learning algorithm that is usually used for binary classification tasks. This model provides the probability $p(y=1 \mid x)$, that is, the probability of a positive result $y=1$ under the given data $x$. LR has the advantage of fast training, and discrete and continuous variables can be used as inputs (33). 
SVM-based classification models are widely used supervised classification algorithms. For a given set of feature data from two groups of patients, SVM attempts to determine the maximum hyperplane between the two classes to maximize its distance to the nearest data points on each side (the so-called support vectors). SVM represents a powerful method fable to obtain good classification results by using only a few data points (34). In particular, if the two samples are not linearly separable, some kernel functions can be used to map them to higher dimensional space where they become more separable (35). In this study, we used four kernel types, including linear, power, sigmoid and radial basis function (RBF) kernels. To maximize the margin distance between the hyperplane and the closest samples of both classes, the internal parameter of the training process, the cost $\mathrm{C}$, was set to 1 after a tuning process to obtain the optimal classification results.

Finally, the final features were used as input for a classification and regression tree analysis (CART) (36) to visually stratify patients into a risk group model to stratify patients into plaque risk groups. The representation for the CART model is a binary tree; each root node represents a single input feature and a split point on that feature. The leaf nodes of the tree contain an output variable that is used to make a prediction. The best splits were identified by the Gini impurity (GI) index:

$$
G I=1-\sum_{i=1}^{n} \mathrm{p}_{\mathrm{i}}^{2}
$$

where $p_{i}$ is the fraction of items in the class $i$.

The models were cross-validated using 5 -fold cross validation (37). This is a resampling method that randomly partitions each feature dataset into five equally sized subsets of samples (folds), maintaining a balanced amount of both classes in each fold. In this way, five models were trained and tested; each of the five folds was used once as the test set, while the four remaining folds were used to train the model. The process was repeated ten times aiming to reduce the variance of the cross validation results and to reduce the unlikely chance of obtaining overoptimistic results with just one run.

The performance of the models (i.e. the ability of radiomics features to discriminate and classify plaques was assessed using receiver operator characteristic curves (ROCs) and the area under the curve (AUC). In addition, class-specific accuracy, precision, recall and F-measure evaluation metrics were also used for their ability to provide classifier output quality. The accuracy is defined as the proportion of correct predictions (both true positives and true negatives) among the total number of cases examined. The precision is defined as the number of true positive results divided by the number of all positive results, including those not identified correctly (it is also known as positive predictive value). It can be thought of as a measure of classifier exactness. A low precision indicates a large number of false positives. The recall is defined as the number of true positive results divided by the number of all samples that should have been identified as positive (it is also known as sensitivity in binary classification or true positive rate). It can be thought of as a measure of classifier completeness. A low recall indicates many False Negatives. The F-score is defined as the harmonic mean of the precision and recall. Similar to AUC, these measures range from 0 to 1, with higher values indicating better classification performance. 


\section{Statistical analysis}

All statistical analyses were performed using the XLSTAT statistical packages (Addinsoft, New York, USA).

\section{Results}

\section{Patient characteristics}

Thirty patients (19 males and 11 females, median age 73 , range 50-86) were included in the study. Patients characteristics are listed in Table 1: 
Table 1

Patient characteristics. Data are presented as the mean \pm SD (standard deviation) or number (\%).US: ultrasound color Doppler; CAD: coronary artery disease; AAA: abdominal aortic surgery; AOCP: peripheral obliterative arterial disease

\begin{tabular}{|ll|}
\hline PATIENTS & $\mathrm{N}(\%)$ \\
\hline Total & 30 \\
\hline Gender & \\
Male & $19(63.33)$ \\
Female & $11(36.67)$ \\
\hline Age (years) & \\
Median & 72.96 \\
Range & $50-86$ \\
\hline Comorbidities & \\
\hline Hypertension & $28(93.33)$ \\
Valvulopathies & $5(16.66)$ \\
Diabetes type 2 & $9(30)$ \\
Hypercholesterolemia & $17(65.4)$ \\
CAD & $12(46.15)$ \\
AAA & $1(3.3)$ \\
AOCP & $2(6.6)$ \\
Cronic Renal failure & $3(10)$ \\
\hline Total cholesterol, mg/dL & $155.34 \pm 36.5$ \\
\hline HDL cholesterol, mg/dL & $49.8 \pm 14.6$ \\
\hline LDL cholesterol, mg/dL & $79.15 \pm 28.8$ \\
\hline Triglycerides, mg/dL & $113.8 \pm 47.6$ \\
\hline Creatinine, mg/dL & $0.92 \pm 0.26$ \\
\hline Stenosis severity (US) & $71.1 \pm 15.3$ \\
\hline Stenosis severity (AngioCT) (\% Nascet) & $80.2 \pm 9.08$ \\
\hline Plaque morphology (surgery) & \\
Soft-Atheromatous debris or intraplaque hemorrhage & $14.3)$ \\
\hline Hard-Calcified or with collagen-rich sclerotic tissue & \\
\hline
\end{tabular}


A total of 230 radiomics features were extracted from each plaque to predict plaque composition.

Pairwise Spearman correlation between features reported a high level of correlations, with more than $80 \%$ correlating with at least one other feature at $|\rho|>0.8$. Furthermore, in univariate analysis, 10 features were found to be significantly associated with the two plaque groups $(p<0.05)$. Detailed diagnostic accuracy statistics for the ten highly correlated features are reported in Table 2.

Table 2

Diagnostic accuracy statistics for the ten highly correlated features

\begin{tabular}{|c|c|c|c|c|c|c|}
\hline & AUC (Cl95\%) & Sensitivity & Specificity & PPV & NPV & Precision \\
\hline \multicolumn{7}{|l|}{$\begin{array}{l}\text { Intensity-based } \\
\text { features }\end{array}$} \\
\hline Entropy & $\begin{array}{l}0,924(0,864- \\
0,984)\end{array}$ & 1.000 & 0.750 & 0.778 & 1.000 & 0.867 \\
\hline \multicolumn{7}{|l|}{$\begin{array}{l}\text { Morphological } \\
\text { features }\end{array}$} \\
\hline Surface & $\begin{array}{l}0.920(0.856- \\
0.983)\end{array}$ & 0.929 & 0.813 & 0.813 & 0.929 & 0.867 \\
\hline Volume & $\begin{array}{l}0.960(0.960- \\
0.960)\end{array}$ & 1.000 & 0.813 & 0.824 & 1.000 & 0.900 \\
\hline Lmajor & $\begin{array}{l}0.893(0.810- \\
0.976)\end{array}$ & 0.857 & 0.813 & 0.800 & 0.867 & 0.833 \\
\hline \multicolumn{7}{|l|}{ GLRLM features } \\
\hline Short run emphasis & $\begin{array}{l}0.799(0.654- \\
0.944)\end{array}$ & 0.786 & 0.750 & 0.713 & 0.800 & 0.767 \\
\hline Long run emphasis, & $\begin{array}{l}0.772 \text { (0.618- } \\
0.927)\end{array}$ & 0.857 & 0.625 & 0.667 & 0.833 & 0.733 \\
\hline Gray level variance & $\begin{array}{l}0.795(0.643- \\
0.946)\end{array}$ & 0.786 & 0.813 & 0.786 & 0.813 & 0.800 \\
\hline \multicolumn{7}{|l|}{ GLSZM features } \\
\hline Small zone imphasis & $\begin{array}{l}0.813(0.677- \\
0.948)\end{array}$ & 0.857 & 0.688 & 0.706 & 0.846 & 0.767 \\
\hline Large zone emphasis & $\begin{array}{l}0.777 \text { (0.626- } \\
0.928)\end{array}$ & 0.857 & 0.625 & 0.667 & 0.833 & 0.733 \\
\hline Zone percentage & $\begin{array}{l}0.795(0.651- \\
0.939)\end{array}$ & 0.786 & 0.750 & 0.733 & 0.800 & 0.767 \\
\hline
\end{tabular}

Out of these features, only two features were selected after the stepwise backward elimination procedure, namely, the entropy, defined as: 


$$
\text { Entropy }=\sum_{i=1}^{n} \mathrm{p}(\mathrm{i}) \log _{2}(\mathrm{p}(\mathrm{i}))
$$

which measures the average amount of information required to encode the image values, and the mesh volume is defined as:

$$
V=\sum_{i=1}^{n} \frac{\mathrm{O}_{\mathrm{a}_{\mathrm{i}}} \cdot\left(\mathrm{O}_{\mathrm{b}_{\mathrm{i}}} \times \mathrm{O}_{\mathrm{c}_{\mathrm{i}}}\right)}{6}
$$

$V$ is calculated from the triangular mesh of the ROl; for each face $i$ in the mesh, defined by points ai, bi and $\mathrm{ci}$, the volume $\mathrm{V}$ of the tetrahedron defined by that face and the origin of the image $(0)$ are calculated, and their sum provides the total volume of the ROI.

The box plots for the two top features found to be associated with plaque classification are shown in Figure 1; the diagnostic performance AUCs were 0.924 and 0.960 for and volume, respectively.

Machine learning modeling and performance

Table 3

reports the performance metrics of the classifiers that were trained using the SVM approach on the two selected features. The values are the averages of the performance measures obtained during the iterations. The methodology provided an accuracy, precision, recall and F-score greater than $80 \%$ for all SVM kernel types. The best performance was registered by the SVM classifier with the RBF kernel, with accuracy, precision, recall and F-score equal to 86.7, $92.9,81.3$ and $86.7 \%$, respectively.

\begin{tabular}{|llllll|}
\hline $\begin{array}{l}\text { SVM kernel } \\
\text { types }\end{array}$ & Accuracy & Precision & Recall & $\begin{array}{l}\text { F- } \\
\text { score }\end{array}$ & AUC \\
\hline Linear & 0.800 & 0.813 & 0.813 & 0.813 & 0.951 \\
\hline Power & 0.833 & 0.867 & 0.813 & 0.839 & 0.951 \\
\hline RBF & 0.867 & 0.929 & 0.813 & 0.867 & 0.964 \\
\hline Sigmoid & 0.800 & 0.813 & 0.813 & 0.813 & 0.942 \\
\hline
\end{tabular}

Table 3: Performance metrics of the four SVM classifiers 
Table 4

shows the outcomes of SVM in terms of confusion matrices. The values listed with a blue background indicate the correct classification of plaques. The importance of this plaque classification is to reduce the negative predictions (including false positives and false negatives). From the confusion matrices, we found that all SVM kernels were able to correctly classify 13 out of 16 hard plaques and that the SVM model using the RBF kernel obtained the best performance for soft plaque classification (only 1 soft plaque was misclassified).

\begin{tabular}{|llllll|}
\hline a) & from $\backslash$ to & Hard & Soft & Total & \% correct \\
\hline & Hard & 13 & 3 & 16 & 81.3 \\
\hline & Soft & 3 & 11 & 14 & 78.6 \\
\hline & Total & 16 & 14 & 30 & 80.0 \\
\hline b) & from $\backslash$ to & Hard & Soft & Total & \% correct \\
\hline & Hard & 13 & 3 & 16 & 81.3 \\
\hline & Soft & 2 & 12 & 14 & 85.7 \\
\hline & Total & 15 & 15 & 30 & 83.3 \\
\hline c) & from $\backslash$ to & Hard & Soft & Total & $\%$ correct \\
\hline & Hard & 13 & 3 & 16 & 81.3 \\
\hline & Soft & 1 & 13 & 14 & 92.9 \\
\hline & Total & 14 & 16 & 30 & 86.7 \\
\hline d) & from $\backslash$ to & Hard & Soft & Total & $\%$ correct \\
\hline & Hard & 13 & 3 & 16 & 81.3 \\
\hline & Soft & 3 & 11 & 14 & 78.6 \\
\hline & Total & 16 & 14 & 30 & 80.0 \\
\hline
\end{tabular}

Table 4:: Confusion matrices of SVM models (a) SVM linear kernel, (b) SVM power kenel, (c) SVM RBF kernel and d) SVM sigmoid kernel

Figure 2 shows the CART classification tree for the two most informative radiomic features. For each node, it was reported the number of objects at each node, the corresponding percentage, the improvement which correspond to the number of observations in the node times, the reduction in impurity caused by the split of the node, the purity that indicates what is the percentage of objects that belong to the dominating category of the dependent variable at this node, the split variable and the value(s) or intervals of the latter, and the class predicted by the node. 
Node 1 represents the initial distribution of the plaques before any criteria had been applied. Nodes 2 and 3 reflect the effect of introducing the volume feature as a first decision criterion. The category volume $\leq$ 481.8 relocates most of the plaques to node 2 with a purity index of $100 \%$, e.g., all plaques have a calcific composition. The plaques in node 3 are located in the soft group and will be subjected to the second criterion for further segregation, e.g., the entropy feature. At this second decision level, entropy values greater than 12.5 relocate all plaques in the soft group with $100 \%$ purity. Looking at Figure 2, the rule built by the algorithm can be expressed in natural language as "If Volume $<481.8$ then plaque $=1$ in $100 \%$ of cases". Similarly, at node 5, "If Entropy $\otimes 12.5$ and Volume $\otimes 481.8$, then plaque $=2$ in $100 \%$ of cases".

The confusion matrix of the results is reported in Table 5 and was used to calculate the percentage of well-classified observations. The model achieved $86.7 \%$ well-classified plaques and an AUC of 0.987 .

Table 5

Confusion Matrix of the CART model

\begin{tabular}{|lllll|}
\hline from $\backslash$ to & Hard & Soft & Total & \% correct \\
\hline Hard & 15 & 1 & 16 & 93.8 \\
\hline Soft & 3 & 11 & 14 & 78.6 \\
\hline Total & 18 & 12 & 30 & 86.7 \\
\hline
\end{tabular}

\section{Discussion}

In recent years, the assessment of carotid atherosclerosis and risk stratification have undergone a paradigm shift moving from the usual measurement of carotid stenosis degree to a more complete characterization of plaque composition. Prospective multicenter trials, such as PARISK (38), confirmed this approach, highlighting the role of plaque morphology in patient outcome. However, plaque imaging techniques still do not representthe basics for clinical decision making and routine clinical practice (39). Recent guidelines have been issued by the European Society for Vascular Surgery (ESVS), aiming to provide a more unified approach for patients with carotid disease (40) and highlighting the need for new clinical/imaging noninvasive reliable methods able to identify the morphological features of vulnerable plaques.

Recently, following this suggestion, several researchers have attempted a radiomics-based methodology to overcome the limitation of visual assessment and provide a more objective method to characterize carotid atherosclerotic plaques (41-43). Zaccagna et al. (41) assessed the potential role of CT texture analysis in identifying vulnerable patients with carotid artery atherosclerosis. The authors highlighted the feasibility of identifying vulnerable patients in stroke and TIA, reporting CT texture as a reliable risk stratification tool in patients with carotid atherosclerosis. In particular, the authors identifiedskewness as the most statistically significant metric able to differentiate between symptomatic and asymptomatic 
patients. Le et al (42) assessed the robustness of carotid artery CT angiography radiomics in the identification of culprit lesions in cerebrovascular events. The authors identified a set of radiomics features that are robust, nonredundant and have superior predictive performance for the classification of culprit versus nonculprit carotid arteries in patients with stroke and TIA. Finally, Zhang et al. (43) successfully implemented a high-risk plaque MRI-based model using radiomics features and machine learning for differentiating symptomatic from asymptomatic carotid plaques. This MRI-based radiomics model was found to be superior to the traditional model in the identification of high-risk plaques.

In this study, we sought to build a machine learning-based model using radiomics features for differentiating hard from soft carotid plaques using CTA imaging. In particular, after the feature extraction and selection processes, the volume and entropy were found to have significantly higher values in plaques considered "soft". Radiomics modeling presents a distinct advantage over "black-box" techniques such as deep learning because it allows a potential interpretability and explanation of the obtained results.In this way, we can attempt to provide enlightenment on the prominence of volume and entropy features in the present classification model. The volume ofcarotid artery plaque has already been proven to be an important determinant of plaque vulnerability and the risk of rupture, and some authors have suggested that plaque volume should be considered a better descriptor of the severity of atherosclerotic disease than the degree of stenosis (44).Saba et al. (45) reported that the lipid component volume is associated with the presence of plaque ulcerations, which represent a significant risk factor for the development of cerebrovascular events, and that there is an increase in the proportion of lipids and calcifications with increasing plaque volume. Recently, Lu et al. (46) reported that the progression of carotid plaque volume is independently associated with recurrent ischemic cerebrovascular events. Therefore, the plaque volume assessment and the monitoring of its temporal changes may improve the ability to estimate risk. The entropy feature describes the complexity of a segmented ROI so that the larger the entropy is, the more complex the ROI. This feature was found to be promising in oncoradiomics, where it has been associated with tumor gene expression, tumor metabolism, tumor stage, patient prognosis, and treatment response $(47,48)$. Since entropy is a measure of texture irregularity, this feature can be a reliable surrogate for the quantification of plaque spatial heterogeneity. In this study, we reported that entropy values were significantly higher for "soft" plaques than for "hard" plaques. This is an indication that soft plaques present a more random density distribution, probably due to the presence of various components with various low-density Hounsfield numbers (HU). In particular, $\mathrm{HU}$ values less than 0 in the plaque are associated with the presence of intraplaque hemorrhage, while $\mathrm{HU}$ values ranging from 0 to 60 are usually associated with fatty plaques (49). Both situations are associated with an increased risk of stroke (50). In contrast, calcified plaques are classified according to $\mathrm{HU}$ values greater than 130 (49), and the presence of calcium seems to represent a protective factor, providing stability against biomechanical stress and disruption (51).

Using these top two ranked features, we trained radiomics-based machine learning models using SVM and CART analysis methods. Among the various models, our results reported that the SVM classifier with the RBF kernel achieved the best performance and was able to successfully create the hyperplane and maximize the margins between the support vectors. It reported higher accuracy and F-score values, both 
equal to $86.7 \%$, able to correctly classify 13 out 16 hard plaques and 13 out 14 soft plaques. We also build a CART model as a knowledge-discovery tool because of its well-known interpretability in terms of variable thresholds. To obtain a simple and small decision tree, we chose to model our CART with only 5 nodes and two depth levels. CART analysis chose patients with a plaque volume greater than 481.8 to be associated with soft plaques, i.e., plaques at increased risk. The entropy feature then subsequently played a significant role in increasing the classification rate among these plaques. The model provided a very high diagnostic performance of $A U C=0.987$; due to the high classification accuracy, we believe that this approach could be used as a valuable adjunct tool for vascular surgeons in prescribing effective treatment options. In particular, for asymptomatic patients with stenosis degrees greater than $70 \%$, the use of a decision tree could provide a valuable tool in discriminating patients eligible for elective surgery. The authors are currently gathering more data to better stratify the plaque classification beyond the proposed binary classification.

Despite encouraging results, the present study has several limitations, and some caution must be employed regarding any robust conclusions. First, the sample size used in this study to train the radiomics analysis of carotid plaques was relatively small $(n=30)$. For this reason, we choose a simple binary plaque classification (hard vs. soft) to allow a reasonable sample size for each group. Second, carotid plaques were from a single center, and radiomics results were based on CT images from one scanner and software analysis system; therefore, generalizability of the results beyond these settings must be proven. Third, although all the radiomics feature calculations were automated, plaque segmentation was manually performed by a senior radiologist, a procedure always prone to interoperator variability. The application of automated segmentation, as already implemented for the definition of volumes in radiation oncology, will reduce the interoperator variability and improve the clinical feasibility of radiomics. Overall, the present study is a pilot study conducted on a smaller scale to assess the ability of a quantitative radiomics approach to differentiate potentially vulnerable plaques. Although reliable internal validation was performed to minimize sample bias with a $\mathrm{k}$-fold cross validation process, the developed model has not been externally validated. An external validation of our results is ongoing with an independent dataset to determine the prediction model's reproducibility and generalizability to new and different patients.

\section{Conclusions}

A radiomics-based analysis of carotid plaques on CTA images can provide a more accurate plaque risk stratification and distinguish between hard and soft plaques. This quantitative approach could be easily implemented in current standard clinical decision paths, increasing the reproducibility andaccuracy of CTA imaging. Prospective larger studies are needed to further ascertain the ability of radiomics to predict future stroke risk.

\section{Declarations}


Funding The authors received no specific financial support for the research, authorship, and/or publication of this article.

Conflict of interest All authors declare no competing interests.

Author Contributions SC, MM, VV and PM contributed to the conception and design of the study. GM, FD, AR and LB organized the database. Sc, JL, EHT amd LI performed the radiomics analysis. LP, MF and CS performed statistical analysis. AP performed radiological analysis. SC, GM and FD wrote the first draft of the manuscript. AP, PM and CS wrote sections of the manuscript. CMDF, EC, VV and MM critically revised the manuscript. All authors contributed to manuscript revision and read and approved the submitted version.

Ethical standards All procedures performed in studies involving human participants or on human tissue were in accordance with the ethical standards of the institutional and/or national research committee and with the 1975 Helsinki declaration and its later amendments or comparable ethical standards. Patients signed informed consent to the treatment procedure; the study was retrospective.

\section{References}

1. Ooi, Y. C. \& Gonzalez, N. R. Management of extracranial carotid artery disease. Cardiol Clin, 33, 1-35 (2015).

2. Rothwell, P. M. et al. Analysis of pooled data from the randomized controlled trials of endarterectomy for symptomatic carotid stenosis., 361, 107-116 (2003).

3. Redgrave, J. N., Lovett, J. K., Gallagher, P. J. \& Rothwell, P. M. Histological assessment of 526 symptomatic carotid plaques in relation to the nature and timing of ischemic symptoms: the Oxford plaque study., 113, 2320-2328 (2006).

4. Pletsch-Borba, L. et al. Change in carotid plaque components: a 4-year follow-up study with serial MR imaging. JACC Cardiovasc Imaging, 11, 184-192 (2018).

5. Zavodni, A. E. et al. Carotid artery plaque morphology and composition in relation to incident cardiovascular events: the multiethnic study of atherosclerosis (MESA)., 271, 381-389 (2014).

6. Zhao, X. Q. et al. Clinical factors associated with high-risk carotid plaque features as assessed by magnetic resonance imaging in patients with established vascular disease (from the AIM-HIGH study). Am J Cardiol, 114, 1412-1419 (2014).

7. Schindler, A. et al. Prediction of stroke risk by detection of hemorrhage in carotid plaques: metaanalysis of individual patient data. JACC Cardiovasc Imaging, 13 (2 Pt 1), 395-406 (2020).

8. Gupta, A. et al. Carotid plaque MRI and stroke risk: a systematic review and meta-analysis., 44, 3071-77 (2013).

9. Saba, L. et al. Imaging biomarkers of vulnerable carotid plaques for stroke risk prediction and their potential clinical implications. Lancet Neurol, 18, 559-572 (2019). 
10. Howard, D. P. et al. Histological features of carotid plaque in patients with ocular ischemia versus cerebral events., 44, 7349 (2013).

11. Finn, A. V. et al. Concept of vulnerable/unstable plaque. Arterioscler Thromb Vasc Biol, 30, 128292 (2010).

12. Saba, L. et al. Imaging of the carotid artery vulnerable plaque. Cardiovasc Intervent Radiol, 37, 572585 (2014).

13. Aboyans, V. et al. ESC Guidelines on the Diagnosis and Treatment of Peripheral Arterial Diseases, in collaboration with the European Society for Vascular Surgery (ESVS): Document covering atherosclerotic disease of extracranial carotid and vertebral, mesenteric, renal, upper and lower extremity arteries. The Task Force for the Diagnosis and Treatment of Peripheral Arterial Diseases of the European Society of Cardiology (ESC) and of the European Society for Vascular Surgery (ESVS). Eur. Heart J, 39, 763-816 (2018).

14. Gupta, A. et al. Detection of symptomatic carotid plaque using source data from MR and CT angiography: a correlative study. Cerebrovasc Dis, 39, 151-161 https://doi.org/10.1159/000373918 (2015).

15. U-King-Im, J. M. et al. Characterization of carotid plaque hemorrhage: a CT angiography and mr intraplaque hemorrhage study., 41, 1623-1629 https://doi.org/10.1161/STROKEAHA.110.579474 (2010).

16. van den Bouwhuijsen, Q. J. et al. Coexistence of calcification, intraplaque hemorrhage and lipid core within the asymptomatic atherosclerotic carotid plaque: the Rotterdam study. Cerebrovasc Dis, 39, 319-324 https://doi.org/10.1159/000381138 (2015).

17. Mosleh, W. et al. High-Risk Carotid Plaques Identified by CT-Angiogram can Predict Acute Myocardial Infarction. Int J Cardiovasc Imaging, 33 (4), 561-568 (2017).

18. Gillies, R. J., Kinahan, P. E. \& Hricak, H. Radiomics: images are more than pictures, they are data., 278, 563-577 https://doi.org/doi: 10.1148/radiol.2015151169 (2016).

19. Liu, Z. et al. The Applications of Radiomics in Precision Diagnosis and Treatment of Oncology: Opportunities and Challenges. Theranostics, 9 (5), 1303-1322 (2019).

20. Seetharam, K., Brito, D., Farjo, P. D. \& Sengupta, P. P. The Role of Artificial Intelligence in Cardiovascular Imaging: State of the Art Review. Front Cardiovasc Med, 23, 7:618849 https://doi.org/doi: 10.3389/fcvm.2020.618849 (2020).

21. Kolossváry, M., De Cecco, C. N., Feuchtner, G. \& Maurovich-Horvat, P. Advanced atherosclerosis imaging by CT: Radiomics, machine learning and deep learning. J Cardiovasc Comput Tomogr, 13 (5), 274-280 (2019).

22. Acharya, U. R. et al. Computed tomography carotid wall plaque characterization using a combination of discrete wavelet transform and texture features: a pilot study. Proc Inst Mech Eng H, 227, 643-654 (2013).

23. Kakkos, S. K. et al. Texture analysis of ultrasonic images of symptomatic carotid plaques can identify those plaques associated with ipsilateral embolic brain infarction. Eur J Vasc Endovasc 
Surg, 33, 422-429 (2007).

24. Zhang, R. et al. Identification of high-risk carotid plaque with MRI-based radiomics and machine learning. Eur. Radiol, https://doi.org/doi: 10.1007/s00330-020-07361-z (2020).

25. Lee, E. P. et al. Assessing robustness of carotid artery CT angiography radiomics in the identification of culprit lesions in cerebrovascular events. Sci. Rep, 11 (1), 3499 https://doi.org/10.1038/s41598021-82760- w (2021).

26. van Assen, M. et al. Automated plaque analysis for the prognostication of major adverse cardiac events. Eur. J. Radiol, 116, 76-83 https://doi.org/10.1016/j.ejrad.2019.04.013 (2019).

27. Zaccagna, F. et al. CT texture-based radiomics analysis of carotid arteries identifies vulnerable patients: a preliminary outcome study., 63 (7), 1043-1052 https://doi.org/doi: 10.1007/s00234-02002628-0 (2021).

28. Barnett, H. J. M. et al. Beneficial effect of carotid endarterectomy in symptomatic patients with highgrade carotid stenosis. N Engl J Med, 325, 445-453 (1991).

29. Avril, G. et al. Carotid endarterectomy plaques: correlations of clinical and anatomic findings. Ann Vasc Surg, 5, 50-4 (1991).

30. Falk, E., Shah, P. K. \& Fuster, V. Coronary plaque disruption., 92, 657-671 (1995).

31. Dinapoli, N. et al. Moddicom: a complete and easily accessible library for prognostic evaluations relying on image features. Annu Int Conf IEEE Eng Med Biol Soc, 771-4 https://doi.org/doi: 10.1109/EMBC.2015.7318476 (2015).

32. Zwanenburg, A. et al. The image biomarker standardization initiative: standardized quantitative radiomics for high-throughput image-based phenotyping., 295, 328-338 (2020).

33. Forghani, R. et al. Radiomics and artificial intelligence for biomarker and prediction model development in oncology. Comput Struct Biotechnol J, 17, 995-1008 https://doi.org/doi: 10.1016/j.csbj.2019.07.001 (2019).

34. Huang, S. et al. Applications of support vector machine (svm) learning in cancer genomics. Cancer Genomics Proteomics, 15, 41-51 https://doi.org/doi: 10.21873/cgp.20063 (2018).

35. David, V. \& Sanchez, A. Advanced support vector machines and kernel methods. Neurocomputing, 55, 5-20 (2003).

36. Breiman, L., Friedman, J. H., Olshen, R. A. \& Stone, C. J. Classification and regression trees (Wadsworth statistics/probability) (Chapman \& Hall, Boca Raton, 1984).

37. Arlot, S. \& Celisse, A. A survey of cross-validation procedures for model selection. Stat. Surv, 4, 40-79 (2010).

38. Truijman, M. T. B. et al. Plaque At RISK (PARISK): prospective multicenter study to improve diagnosis of high-risk carotid plaques. Int J Stroke, 9 (6), 747-54 https://doi.org/doi: 10.1111/ijs.12167 (2014).

39. Saba, L. et al. Imaging of the Carotid Artery Vulnerable Plaque. Cardiovasc Intervent Radiol, 37, 572585 (2014). 
40. Naylor, A. R. et al. Management of atherosclerotic carotid and vertebral artery disease: 2017 Clinical Practice Guidelines of the European Society for Vascular Surgery (ESVS). Eur J Vasc Endovasc Surg, 55, 3-81 https://doi.org/10.1016/j.ejvs.2017.06.021 (2017).

41. Zuccagna, F. et al. CT texture-based radiomics analysis of carotid arteries identifies vulnerable patients: a preliminary outcome study., 63 (7), 1043-1052 https://doi.org/doi: 10.1007/s00234-02002628-0 (2021).

42. Le, E. P. V. et al. Assessing robustness of carotid artery CT angiography radiomics in the identification of culprit lesions in cerebrovascular events. Scientifc Reports, 11, 3499 https://doi.org/10.1038/s41598-021-82760-w (2021).

43. Zhang, R. et al. Identification of high-risk carotid plaque with MRI-based radiomics and machine learning. Eur Radiol, 31, 3116-3126 (2021).

44. Ouhlous, M., Flach, H. Z. \& de Weert, T. T. Carotid plaque composition and cerebral infarction: MR imaging study. Am J Neuroradiol, 26, 1044-1049 (2005).

45. Saba, L. et al. Association between carotid artery plaque volume, composition, and ulceration: a retrospective assessment with MDCT. AJR Am J Roentgenol, 199, 151-15670 (2012).

46. Lu, M. et al. Association of progression of carotid artery wall volume and recurrent transient ischemic attack or stroke: a magnetic resonance imaging study., 49, 614-620 (2018).

47. Yip, C. et al. Primary esophageal cancer: heterogeneity as potential prognostic biomarker in patients treated with definitive chemotherapy and radiation therapy., 270, 141-148 https://doi.org/doi:10.1148/radiol.13122869 (2014).

48. Zhang, H. et al. Locally advanced squamous cell carcinoma of the head and neck: CT texture and histogram analysis allow independent prediction of overall survival in patients treated with induction chemotherapy., 269, 801-809 https://doi.org/doi:10.1148/radiol.13130110 (2013).

49. de Weert, T. T. et al. In vivo characterization and quantification of atherosclerotic carotid plaque components with multidetector computed tomography and histopathological correlation. Arterioscler Thromb Vasc Biol, 26 (10), 2366-2372 (2006).

50. Saba, L., Montisci, R., Sanfilippo, R. \& Mallarini, G. Multidetector row CT of the brain and carotid artery: a correlative analysis. Clin Radiol, 64 (8), 767-778 (2009).

51. Nandalur, K. R. et al. Calcified carotid atherosclerosic plaque is associated less with ischemic symptoms than is noncalcified plaque on MDCT. AJR Am J Roentgenol, 184, 295-298 (2005).

\section{Figures}



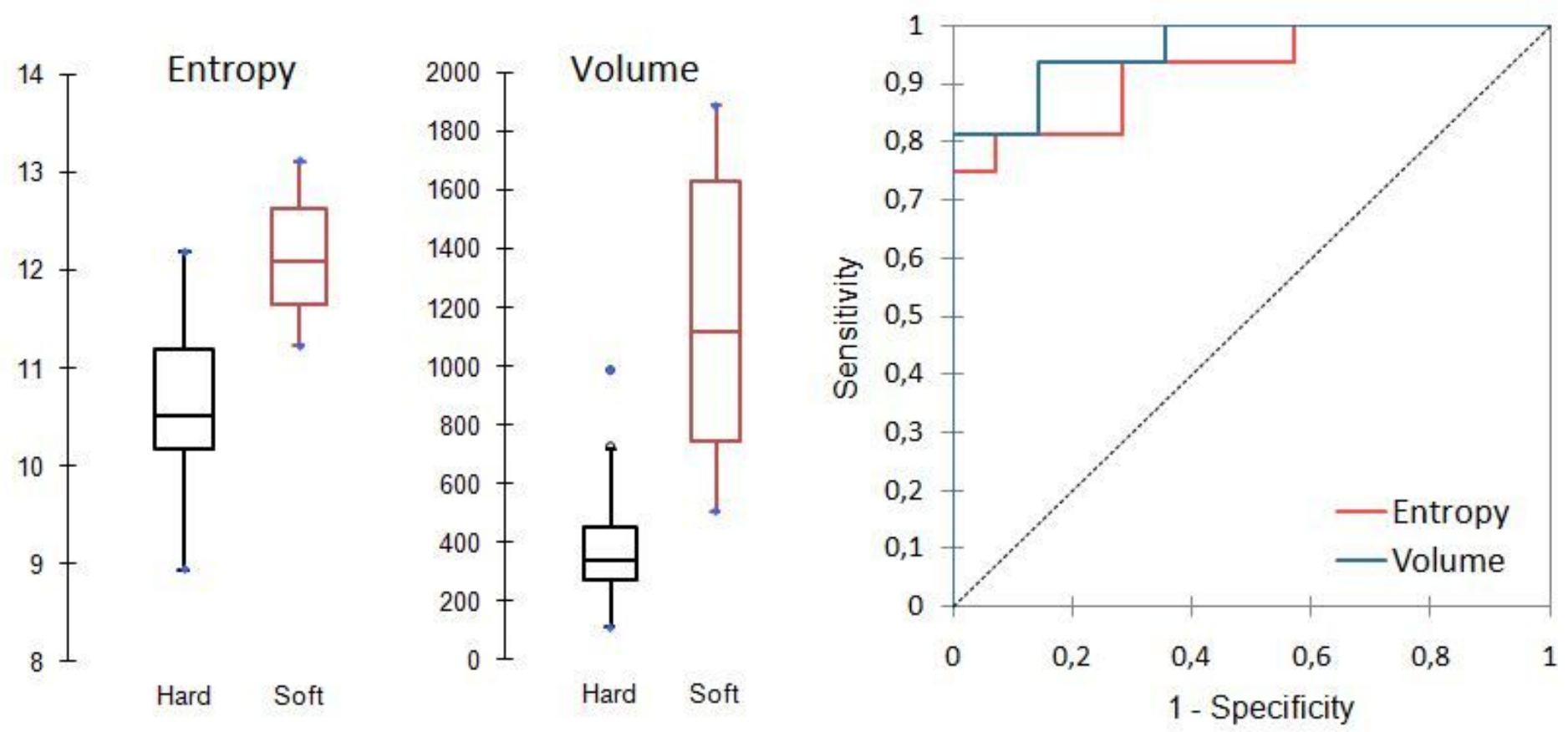

Figure 1

Diagnostic performance of the entropy and volume features for plaque composition prediction. 


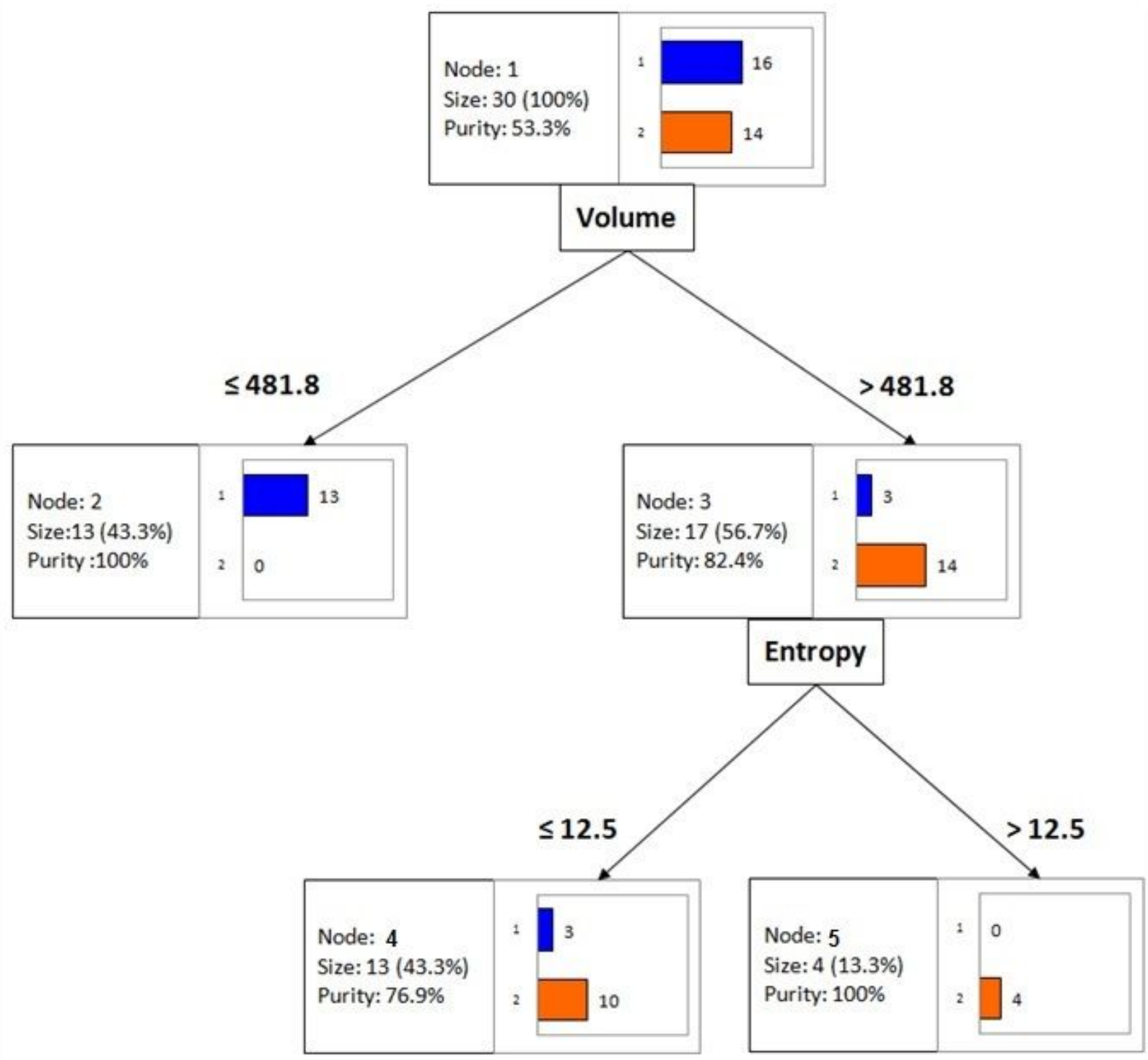

Figure 2

CART classification tree for the volume and entropy features 